\title{
Bilayer Splitting in the Electronic Structure of Heavily Overdoped $\mathrm{Bi}_{2} \mathrm{Sr}_{2} \mathrm{CaCu}_{2} \mathrm{O}_{8+\delta}$
}

\author{
D. L. Feng, N. P. Armitage, D. H. Lu, A. Damascelli, J. P. Hu, P. Bogdanov, A. Lanzara, F. Ronning, K. M. Shen, \\ H. Eisaki, C. Kim, and Z.-X.Shen \\ Department of Physics, Applied Physics and Stanford Synchrotron Radiation Laboratory, \\ Stanford University, Stanford, CA 94305, USA \\ J.-i. Shimoyama, and K. Kishio \\ Department of Applied Chemistry, University of Tokyo, Tokyo, 113-8656, Japan
}

(Jan. 17, 2000)

\begin{abstract}
The electronic structure of heavily overdoped $\mathrm{Bi}_{2} \mathrm{Sr}_{2} \mathrm{CaCu}_{2} \mathrm{O}_{8+\delta}$ is investigated by angle-resolved photoemission spectroscopy. The long-sought bilayer band splitting in this two-plane system is observed in both normal and superconducting states, which qualitatively agrees with the bilayer Hubbard model calculations. The maximum bilayer energy splitting is about $88 \mathrm{meV}$ for the normal state feature, while it is only about $20 \mathrm{meV}$ for the superconducting peak. This anomalous behavior cannot be reconciled with the quasiparticle picture.
\end{abstract}

PACS numbers: 71.18.+y, 74.72.Hs, 79.60.Bm

High temperature superconductors (HTSC's), as doped Mott insulators, show strong doping dependent behavior. The underdoped regime of the HTSC's is characterized by its unconventional properties, such as the pseudogap and non-Fermi liquid transport behavior. On the other hand, the overdoped regime is considered to be more "normal", partly because of the absence of a pseudogap and more Fermi liquid-like behavior. It is very challenging and important for HTSC theories to be able to explain the phenomenology in both regimes. Angle resolved photoemission spectroscopy (ARPES), one of the most direct probes of the electronic structure, has contributed greatly to the understanding of the electronic structure of the HTSC's. However, most systems studied by ARPES have either low $T_{c}$ 's (below $40 \mathrm{~K}$ for $\mathrm{La}_{2-x} \mathrm{Sr}_{x} \mathrm{CuO}_{4+\delta}$ (LSCO), and $\mathrm{Bi}_{2} \mathrm{Sr}_{2} \mathrm{CuO}_{6+\delta}(\mathrm{Bi} 2201)$ ), or doping limitations (only up to slightly overdoping for $\mathrm{Bi}_{2} \mathrm{Sr}_{2} \mathrm{CaCu}_{2} \mathrm{O}_{8+\delta}$ (Bi2212) and $\left.\mathrm{YBa}_{2} \mathrm{Cu}_{3} \mathrm{O}_{7-y}(\mathrm{YBCO})\right)$. For a complete understanding, it is very important to study the heavily overdoped systems, especially Bi2212, which is the most studied system by ARPES.

Recent advances in high pressure annealing techniques have made it possible to synthesize heavily overdoped Bi2212. In this paper, we report ARPES measurements of the electronic structure of heavily overdoped Bi2212. We show that the long-sought bilayer band splitting (BBS) exists for both normal and superconducting states of this material over large fraction of the Brillouin zone. The detection of the BBS, which has been predicted by band structure calculations [1,2], but not observed in earlier ARPES data [3], enables us to address several important issues. First, it provides a very detailed test for the theoretical calculations, with our experimental results favoring the bilayer Hubbard model 画 over LDA calculations [1.2]. Second, it shows the novel result that the bilayer splitting energy in the superconducting state is only about $23 \%$ of the normal state splitting. Third, it provides an explanation for the detection of a "peak-diphump" structure in the normal state of heavily overdoped samples [5.,6].

Heavily overdoped Bi2212 samples $\left(T_{C}\right.$ (onset) $=65 \mathrm{~K}$, $\Delta T_{C}(10 \% \sim 90 \%)=3 \mathrm{~K}$, denoted as OD65) were synthesized by annealing floating-zone-grown single crystals under oxygen pressure $P_{\mathrm{O}_{2}}=300 \mathrm{~atm}$ at $300^{\circ} \mathrm{C}$ for two weeks, and characterized by various techniques. Magnetic susceptibility measurements show that the presence of a second phase is less than $1 \%$. Laue diffraction and low energy electron diffraction (LEED) patterns show that its superstructure and surface resemble those of optimally doped samples, and the flatness of the cleaved sample surface is shown by the small laser reflection from the sample. Angle resolved photoemission experiments were performed at beamline V-4 of Stanford Synchrotron Radiation Laboratory (SSRL) with a Scienta SES200 electron analyzer, which can take spectra in a narrow cut of $0.5^{\circ} \times 14^{\circ}$ simultaneously in its angular mode with an angular resolution as good as $0.12^{\circ}$ along the cut direction. The data were collected with polarized synchrotron light from a normal incidence monochromator, where the intensity of the second order light is extremely weak, as well as He-I light from a He discharge lamp. The overall energy resolution varied from $10 \mathrm{meV}$ to $17 \mathrm{meV}$ under different conditions. The chamber base pressure was better than $5 \times 10^{-11}$ torr during the measurements.

ARPES spectra were taken over a wide region of the Brillouin zone of OD65. Fig. 1 1a-h show the normal state photoemission intensity as a function of momentum and binding energy in false color. In this way, one can clearly see the centroids of the dispersing features. For example, Fig. Ia shows that one band disperses and crosses the Fermi energy along a momentum cut that goes through 
the $d$-wave node region. Away from the nodal region, this seemingly single feature splits into two features, Features $\mathrm{A}$ and B, starting from Fig. 11c. The photoemission intensities in the bracketed region are replotted in the form of energy distribution curves (EDC's) in Fig. 1 i i, where spectra show that two peaks that cross the Fermi level less than $0.6^{\circ}$ apart. This splitting increases when approaching the $(\pi, 0)$ region. In Fig. Th, the Features A and B are well-separated, and two more weaker features are clearly visible as well; these are the superstructure images of Features A and B, which are typically about $(0.22 \pi, 0.22 \pi)$ away from their corresponding main features in Bi2212. The absence of splitting in the nodal region is checked with the best achievable angular resolution $\left(\sim 0.12^{\circ}\right)$.

The observed two bands in the normal state spectra of Bi2212 samples can be naturally interpreted by the presence of the BBS. Because the Bi2212 ARPES features are considered to be mainly contributed by the anti-bonding $x^{2}-y^{2}$ state in the $\mathrm{CuO}_{2}$ plane, and $\mathrm{Bi} 2212$ has two $\mathrm{CuO}_{2}$ planes per unit cell, the intrabilayer coupling would cause a splitting. As we will see later, the observed splitting agrees with what is expected from a bilayer system [ $[1$ ]. This interpretation is also supported by recent studies of heavily overdoped single-layer Bi2201, where only one band was observed [7. Since Feature A is always at lower binding energy than Feature B at a given momentum, we assign the anti-bonding band $(\mathrm{AB})$ to Feature $\mathrm{A}$, and bonding band (BB) to Feature B.

The Fermi surfaces (FS's) can be determined by determining Fermi crossings of the bands (dispersion method), or determining the local maxima of the low energy ARPES spectral weight distribution (spectral weight method) (Fig. 2) 81. One can see two main FS's, one for the antibonding band $(\mathrm{AB})$ and the other for the bonding band $(\mathrm{BB})$, and their corresponding superstructure images ( $\mathrm{AB}$ ' and $\mathrm{BB}$ '). The observed hole-like Fermi surface topology is consistent with early findings in less overdoped Bi2212 systems at similar photon energies. These FS's overlap in the nodal region and gradually depart from each other when approaching the $(\pi, 0)$ region. Fig. $2 \mathrm{~b}$ shows EDC's along one cut that crosses all of the four Fermi surfaces. At $22.7 \mathrm{eV}$ photon energy (lower right half of Fig. 2a), the $\mathrm{AB}$ has more weight near $E_{F}$ than the $\mathrm{BB}$, and this situation is reversed at $20 \mathrm{eV}$ (upper left half of Fig. 2a). This strong photon energy dependence of the relative intensities of the $\mathrm{AB}$ and $\mathrm{BB}$ is consistent with the $\mathrm{BBS}$, because the $\mathrm{AB}$ and $\mathrm{BB}$ have odd and even symmetries respectively along the c-axis. By tuning the incident photon energy, the wavevector of the final electron state along the c-axis is changed, which changes the photoemission cross-sections between the final state and the initial $\mathrm{BB}$ and $\mathrm{AB}$ differently due to their opposite symmetries. The fact that we see BBS all over the FS and in the superstructure images (AB' and BB') away from the $(\pi, 0)$ region rules out the possibility that the split FS's are artifacts caused by the superstructure. Moreover, because the intensity of $\mathrm{BB}$ is weaker than that of
$\mathrm{AB}$ in the $22.7 \mathrm{eV}$ photon energy data, $\mathrm{AB}$ cannot be a superstructure of $\mathrm{BB}$, and vice versa for the data taken at $20 \mathrm{eV}$ photon energy.

To understand the effect of the BBS on the superconducting state, spectra were taken in both the normal and superconducting states near the $(0, \pi)$ region (Fig. 3), where the splitting is greatest. Moreover, it was found that in this region, the ARPES lineshape of Bi2212 evolves dramatically across $T_{c}$ from a broad spectrum in the normal state into a well-known peak-diphump (PDH) structure in the superconducting state 9.

In the normal state (Fig. 3a), the antibonding state crosses $E_{F}$ near n 4 and n- 4 , while the bonding state disperses through the Fermi energy around spectra n8 and n-8. The presence of two features in the normal state was reported earlier [5.6], and suggested to be an anomalous normal state counterpart to the conventional superconducting PDH [5]. Here, we show that this feature is actually due to the bilayer splitting. In spectra n-3 through $\mathrm{n} 3$, the $\mathrm{BB}$ is at high binding energy and thus broad, while the $\mathrm{AB}$ is at low binding energy and thus sharp, which conspire to give a PDH-like structure. We stress that this is fundamentally different from the PDH structure that turns on at $T_{c}$.

In the superconducting state (Fig. $3 \mathrm{~b}$ ), the low energy part of the spectra evolves into two sharp superconducting peaks. It appears that both the normal state $\mathrm{BB}$ and $\mathrm{AB}$ develop their own superconducting $\mathrm{PDH}$ structure. Similar to the superconducting peak reported before in less overdoped samples, both $\mathrm{BB}$ and $\mathrm{AB}$ superconducting peaks lose their intensity upon crossing the corresponding normal state BB/AB FS's. More specifically, spectra s7 and $\mathrm{n} 7$ (replotted in Fig. 3ic), which consist mainly of the $\mathrm{BB}$, strongly resemble the normal and superconducting state spectra from overdoped samples with less carrier doping [10]. When the BB superconducting peak disperses to higher binding energies, it becomes weaker and presumably contributes very little to the sharp peak seen at s0. Therefore, the observed sharp peak at s0 can be regarded as mainly due to the antibonding state. For spectra containing two peaks, they can be fitted by two PDH's, as shown in Fig. 3c for s4.

The dispersions extracted from Fig. 3 are summarized in Fig. Aa. Because the superconducting peak intensity of the $\mathrm{BB}$ is very weak near $(\pi, 0)$, its position is extrapolated and shown as the dotted line. Although the $\mathrm{BB}$ and $\mathrm{AB}$ superconducting peaks have different dispersions, their minimum binding energies near their respective FS's are almost the same $(\sim 16 \mathrm{meV})$, which shows that the $\mathrm{BB}$ and $\mathrm{AB}$ have the same $d$-wave superconducting gap amplitude. The maximum energy splittings can be extracted from the binding energies at $(\pi, 0)$. They are found to be about $88 \mathrm{meV}$ for the normal state bands, and interestingly, only about $20 \mathrm{meV}$ for the superconducting peaks. The striking difference in the splitting energies cannot be explained conventional theories, where quasiparticles below $T_{c}$ have an energy of $E_{\mathbf{k}}=\sqrt{\Delta_{\mathbf{k}}^{2}+\varepsilon_{\mathbf{k}}^{2}}$, with $\varepsilon_{\mathbf{k}}$ and $\Delta_{\mathbf{k}}$ being the normal 
state quasiparticle energy and superconducting gap, respectively. The small splitting energy of the superconducting peak also counters the naive expectation that global phase coherence below $T_{c}$ will enhance the $c$-axis coupling and thus cause larger splitting. Instead, the data demonstrate a qualitative breakdown of this quasiparticle concept. This conclusion is in concert with the earlier observation that the weight of the superconducting peak is closely related to the carrier doping level and the condensation fraction of the system [10]. We hope the new data can stimulate more theoretical works on this issue.

The nature of the normal state BBS as a function of momentum and energy puts strong constraint on theoretical models. A maximum momentum splitting near $(\pi, 0)$ contradicts early LDA calculations, where the calculated BiO Fermi surface near $(\pi, 0)$ causes very a small splitting of the $\mathrm{CuO}_{2}$ bands near $(\pi, 0)[\mathbb{1}$. However, it does agree with bilayer LDA calculations that only consider bands from the two $\mathrm{CuO}_{2}$ planes [2], and the bilayer Hubbard model, which is based on the bilayer LDA band calculations plus additional on-site Coulomb repulsion [4]. The bilayer Hubbard model predicts two AB/BB Fermi surfaces similar to the data for similar carrier doping levels [4.

The bilayer LDA calculations [2] predicted that the normal state bilayer energy splitting to be $2 t_{\perp}(\mathbf{k})=$ $t_{\perp}\left(\cos \left(k_{x} a\right)-\cos \left(k_{y} a\right)\right)^{2} / 2$, where $t_{\perp}(\mathbf{k})$ is the anisotropic intrabilayer hopping. It indicates that the maximum energy splitting is $2 t_{\perp}$ at $(\pi, 0)$. This agrees with the data, and one obtains the experimental intrabilayer hopping $t_{\perp, \text { exp }}=44 \pm 5 \mathrm{meV}$. To test this over a large momentum range, the normal state energy splitting along the

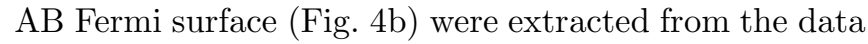
in Fig.'s 11 and 2. Indeed, the data can be fitted very well by $t_{\perp, \exp }\left(\cos \left(k_{x} a\right)-\cos \left(k_{y} a\right)\right)^{2} / 2$, but quantitatively, the experimental maximum energy splitting of $88 \mathrm{meV}$ $\left(2 t_{\perp, \text { exp }}\right)$, is much smaller than the $300 \mathrm{meV}\left(2 t_{\perp, L D A}\right)$ splitting predicted by the bilayer LDA calculations [2]. On the other hand, the data agree better with the bilayer Hubbard model [4], which predicted a similar anisotropic energy splitting with $40 \mathrm{meV}$ maximum energy splitting at $(\pi, 0)$ for the similar doping level. This is because unlike the bilayer LDA calculations, the bilayer Hubbard model considers strong correlations, and strong on-site Coulomb repulsion (or correlations) will substantially reduce the hopping to an occupied site thus reducing the effective intrabilayer hopping. Based on this, its small splitting energy scale (40 meV) may suggest that weaker on-site Coulomb repulsion should be adopted in the bilayer Hubbard model (at least for the heavily overdoped case). We note that $t_{\perp, \exp }$ is of similar magnitude of the gap, and is a significant fraction of the in-plane exchange coupling $J$, and the bandwidth. Therefore, the intrabilayer coupling should be considered in models describing Bi2212.

A natural question is why the bilayer band splitting is particularly prominent in heavily overdoped materials.
This is mainly because the more Fermi liquid-like behavior in the heavily overdoped regime results in much better defined quasiparticles, i.e., much sharper features. The absence of two well-defined features in the spectra of less overdoped samples does not necessarily imply the absence of the BBS. In fact, with improved resolution, preliminary studies have found signatures of BBS in the normal state of slightly overdoped Bi2212 samples 11 .

In summary, the electronic structure of heavily overdoped $\mathrm{Bi}_{2} \mathrm{Sr}_{2} \mathrm{CaCu}_{2} \mathrm{O}_{8+\delta}$ is investigated by angleresolved photoemission spectroscopy. The bilayer band splitting in this two-plane system is observed in both normal and superconducting states, which qualitatively agrees with the bilayer Hubbard model calculations. The different energy splitting scales reported here provide new information for the behavior of the superconducting peak, which cannot be well understood in the quasiparticle framework and needs further investigation.

Stanford Synchrotron Radiation laboratory is operated by the DOE Office of Basic Energy Science Divisions of Chemical Sciences and Material Sciences. The Material Sciences Division also provided support for the work. The Stanford experiments are also supported by the NSF grant 9705210 and ONR grant N00014-98-10195-A00002.

[1] S. Massidda et al., Physica C 152251 (1988); W. E. Pickett, Rev. Mod. Phys. 61 (2), 251 (1989) and references therein.

[2] S. Chakravaty et al., Science 261, 337 (1993); O. K. Anderson et al., J. Phys. Chem Solids 12, 1573 (1995).

[3] H. Ding et al., Phys. Rev. Lett. 76, 1533 (1996).

[4] A. I. Liechtenstein et al.,Phys. Rev. B 54, 12505 (1996).

[5] S. Rast et al. Europhys. Lett. 51 (1), 103 (2000). An abrupt change in the measured ARPES spectra at a new temperature scale $T^{+} \approx 85 \mathrm{~K}$ is also reported. However, it is not observed in our experiments.

[6] Z. Yusof and B. O. Wells, private communication.

[7] D. L. Feng et al., unpublished.

[8] We note that at $20 \mathrm{eV}$ photon energy, the FS's determined by the dispersion method do not agree with those determined by the spectral weight method, since the spectral weight method is affected by the photoemission matrix elements. When two features are very close and have similar intensities, their spectral weight overlaps, and the positions of the local spectral weight maxima shift. However, we stress that the spectral weight method can give a qualitative and objective measurement of the FS with the aid of the dispersion method, which relies on subjective judgement.

[9] D. S. Dessau et al., Phys. Rev. Lett. 66, 2160 (1991).

[10] D. L. Feng et al., Science 289, 277 (2000); H. Ding et al., preprint, cond-matt/0006143.

[11] P. Bogdanov, A. Lanzara, Z.-.X. Shen, unpublished; Y.- 
D. Chung, A. Gromko, D. Dessau, unpublished.

FIG. 1. (color) (a-h) false color scale plot of the OD65 normal state $(\mathrm{T}=75 \mathrm{~K})$ ARPES spectra taken with $22.7 \mathrm{eV}$ synchrotron light, they are along the momentum cuts indicated by lines in the inset. Feature A and B, and their superstructure image A' and B' are indicated by triangles, circles, squares, diamonds respectively. The EDC's near the Fermi crossing in (c) (indicated by "[") are plotted in (i). The angular resolution is $0.3^{\circ}$.

FIG. 2. (color) (a) False color plot of the momentum distribution of the spectral weight near $E_{F}([-20 \mathrm{meV}, 10 \mathrm{meV}])$ of OD65 taken at $22.7 \mathrm{eV}$ (lower right half, $\mathrm{T}=75 \mathrm{~K}$ ) and 20 eV (upper left half, $\mathrm{T}=80 \mathrm{~K}$ ) (note they are from different experiments). The Fermi surface determined by dispersion is also plotted for antibonding states ( $\mathrm{AB}$, triangles), bonding states ( $\mathrm{BB}$, circles), superstructure images of antibonding states (AB', squares), and bonding states (BB', diamonds). (b) ARPES spectra along the cut indicated by the arrow in (a).

FIG. 3. ARPES spectra taken on OD65 with He-I light for (a) normal state, and (b) superconducting state, where the superconducting peak of the antibonding and bonding states are indicated by crosses and bars respectively. The angular resolution is $0.56^{\circ}$. (c) shows selected spectra from (a) and (b). Note that the fit of $\mathrm{s} 4$ is not unique. The spectra are taken along $(-0.24 \pi, \pi)-(0.24 \pi, \pi)$, and labeled from -9 to 9 as shown in the inset of (c).
FIG. 4. (a) Dispersion extracted from Fig. 3 of the superconducting peaks for the bonding sate (bars) and the antibonding state (crosses), and the normal state bands of the bonding sate (solid circles) and the antibonding state (triangles). (b) Energy splitting along the AB Fermi surface, which are obtained from data shown in Fig. 1. It is simply the binding energy of the $\mathrm{BB}$, since the binding energy of $\mathrm{AB}$ is zero at its Fermi surface. The curve is $t_{\perp, \exp }\left[\cos \left(k_{x} a\right)-\cos \left(k_{y} a\right)\right]^{2} / 2$, where $t_{\perp, \exp }=44 \pm 5 \mathrm{meV}$. Error bars are due to the uncertainties in determining the energy position. 
This figure "fig1.jpg" is available in "jpg" format from: http://arxiv.org/ps/cond-mat/0102385v2 
This figure "fig2.jpg" is available in "jpg" format from: http://arxiv.org/ps/cond-mat/0102385v2 
This figure "fig3.jpg" is available in "jpg" format from: http://arxiv.org/ps/cond-mat/0102385v2 
This figure "fig4.jpg" is available in "jpg" format from: http://arxiv.org/ps/cond-mat/0102385v2 